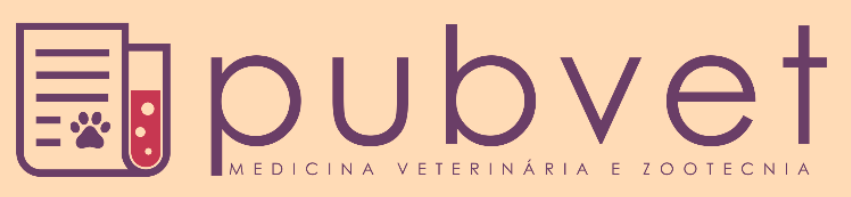

https://doi.org/10.31533/pubvet.v14n3a531.1-8

\title{
Complicações após ruminocentese em bovinos de engorda submetidos à dieta de alto grão isenta de forragem
}

\author{
Angela Maria $\operatorname{Reck}^{1 *} \bullet$, Desiree Vera Pontarolo ${ }^{1} \bullet$, Alessandra Mayer Coelho $^{2} \bullet$, Bruna \\ Artner $^{2} \bullet$, Mikael Neumann ${ }^{\circ}$, , Heloisa Godoi Bertagnon ${ }^{\circ}$ \\ ${ }^{I}$ Discentes do Curso de Pós-Graduação em Ciências Veterinárias, Universidade Estadual do Centro-Oeste, Guarapuava, PR, Brasil. \\ ${ }^{2}$ Discentes do Curso de Medicina Veterinária, Departamento Medicina Veterinária, Universidade Estadual do Centro-Oeste, Guarapuava, PR, Brasil. \\ ${ }^{3}$ Docentes do Departamento de Medicina Veterinária, Universidade Estadual do Centro-Oeste, Guarapuava, PR, Brasil. \\ *Autor para correspondência, E-mail: angelareck@ hotmail.com
}

Resumo. Para potencializar a produtividade de bovinos de corte, utilizam-se cada vez mais dietas a base de grãos que embora forneçam mais energia, alteram o $\mathrm{pH}$ e microbiota ruminal, culminando numa afecção chamada síndrome acidose ruminal sub aguda (SARA). Seu diagnóstico precoce, mediante análises do $\mathrm{pH}$ ruminal, permite intervenções de manejo, impedindo ou minimizando os sinais clínicos. A técnica mais recomendada para esta análise é a ruminocentese, amplamente aplicada em bovinos leiteiros, mas pouco descrita em bovinos de engorda. Portanto, o objetivo deste trabalho foi verificar se esta técnica pode ser aplicada em bovinos de engorda submetidos à dieta $100 \%$ concentrado e isenta de forragens. A ruminocentese foi realizada por punção percutânea do saco ventro caudal de 32 novilhos não castrados (1/2 sangue Angus) de aproximadamente $350 \mathrm{~kg}$ e 11 meses de idade, em dois momentos, M0 momento em que era fornecida dieta composta por volumoso e concentrado e no M1 fornecido dieta $100 \%$ concentrado isenta de forragem. Foram analisadas facilidade de obtenção, características do líquido ruminal e alterações locais nos animais após a punção. No M0 não foi observada alterações locais em nenhum dos animais e no M1 todos os animais apresentaram reações, desde pequenos nódulos subcutâneos até abscessos maiores que $20 \mathrm{~cm}$ de diâmetro. Acredita-se que as complicações após a ruminocentese ocorreram devido ao temperamento dos animais, maior acúmulo de gordura na região da punção e/ou devido às características do líquido ruminal ocasionadas pela dieta, permitindo concluir que a técnica de ruminocentese idealizada para bovinos de leite não foi adequada para bovinos de engorda submetidos à dieta de alto grão isenta de forragens.

Palavras chave: Abscesso, confinamento, novilhos, pH, SARA

\section{Abscess after rumenocentesis in beef cattle subjected to high grain diet}

Abstract. To enhance the productivity of beef cattle, it has been used increasingly diets based on grains, which although they provide more energy, alter the $\mathrm{pH}$ and ruminal microflora, resulting in a condition called subacute ruminal acidosis syndrome (SARA). Early diagnosis of this disease through analysis of ruminal $\mathrm{pH}$, allows it to be established management interventions, preventing or minimizing the clinical signs. The most recommended technique for this analysis is the rumenocentesis, widely applied in dairy cattle, but little described in beef cattle. Therefore, the objective of this study was to determine whether this technique can be applied repeatedly in bulls subjected to high grain diet. The rumenocentesis was performed by percutaneous puncture at caudo ventral sac of 32 young bulls $1 / 2$ Angus blood of approximately $350 \mathrm{~kg}$ and 11 months of age, in two stages, M0 when it was provided diet composed of roughage and concentrate and M1 composed of $100 \%$ concentrate diet. Were analyzed the facility to obtain ruminal fluid, its 
characteristics and local changes. In M0 was not observed local changes in any of the animals, and all animals in M1 had reactions from small subcutaneous nodules to larger abscesses than $20 \mathrm{~cm}$ in diameter. It is believed that complications after rumenocentesis occurred due to animal temper, a higher accumulation of fat in the puncture area and/or due to ruminal fluid characteristics caused by diet, which showed that the rumenocentesis technique devised for cattle milk was not suited to beef cattle subjected to high grain diets.

Keywords: Abscess, cattle feedlot, pH, SARA, steers

\section{Complicaciones después de ruminocentesis en ganado de engorda sometidos a dieta alta en granos y exenta de forraje}

Resumen. Para mejorar la productividad del ganado vacuno, se utilizan dietas cada vez más basadas en granos que, aunque proporcionan más energía, alteran el pH y el microbiota ruminal, culminando en una condición llamada síndrome de acidosis ruminal subaguda aguda (SARA). Su diagnóstico precoz, a través de análisis de $\mathrm{pH}$ ruminal, permite intervenciones de manejo, previniendo o minimizando los signos clínicos. La técnica más recomendada para este análisis es el ruminocentesis, ampliamente aplicado en el ganado lechero, pero poco descrito en el ganado de ceba. Por lo tanto, el objetivo de este trabajo fue verificar si esta técnica se puede aplicar al ganado ceba sometidoa a una dieta $100 \%$ concentrada y libre de forraje. La ruminocentesis se realizó por punción percutánea de la bolsa ventrocaudal de 32 novillos enteros (( $1 / 2$ sangre Angus) de aproximadamente $350 \mathrm{~kg}$ y 11 meses de edad, en dos momentos, M0 - momento en que se proporcionó la dieta compuesta de forraje y concentrado y en M1 - se proporcionó dieta 100\% concentrada libre de forraje. Se analizó la facilidad de obtención, las características del líquido ruminal y los cambios locales en los animales después de la punción. En M0, no se observaron alteraciones locales en ninguno de los animales y en M1 todos los animales presentaron reacciones, desde pequeños nódulos subcutáneos hasta abscesos de más de $20 \mathrm{~cm}$ de diámetro. Se cree que las complicaciones después de la ruminocentesis ocurrieron debido al temperamento de los animales, mayor acumulación de grasa en la región punzante y/o debido a las características del líquido ruminal causado por la dieta, permitiendo concluir que la técnica ruminocentesis idealizada para ganado lechero no fue adecuada para ganado de cebado con una dieta 100 de granos.

Palabras clave: Absceso, confinamiento, novillos, pH, SARA

\section{Introdução}

Os ruminantes foram adaptados a ingerir dietas predominantemente baseadas em forrageiras, absorvendo energia principalmente pela fermentação bacteriana de carboidratos em ácidos orgânicos no rúmen (Berchielli et al., 2011; Van Soest, 1994). No entanto, o crescente mercado consumidor busca potencializar a produtividade animal, utilizando com maior frequência dietas a base de grãos e cereais (Monteschio et al., 2019; Ornaghi et al., 2017; Rivaroli et al., 2017). As dietas compostas por grãos e cereais, embora otimizem a ingestão de energia, podem gerar um quadro de diferente gravidade chamado de síndrome de acidose ruminal subaguda (SARA), uma desordem metabólica, na qual ocorrem episódios diários de $\mathrm{pH}$ ruminal mais baixo, resultado da seleção da microbiota que produz ácidos graxos de cadeia curta fortes como o propiônico e o lático (Noro et al., 2013; Zebeli et al., 2012; Zebeli \& Ametaj, 2009; Zebeli \& Metzler-Zebeli, 2012).

$\mathrm{O}$ pH ruminal, quando próximo à 5.0, promove a destruição principalmente de bactérias gram negativas, que liberam moléculas de sua membrana externa, os lipopolissacarídeos (LPS), que são endotoxinas potencialmente inflamatórias (Cumby et al., 2001; Khafipour et al., 2011; Plaizier et al., 2001). Além disso, este $\mathrm{pH}$ mais baixo também promovem paraqueratose e redução da barreira funcional do rúmen, permitindo que a microbiota ruminal e os LPS alcancem a corrente sanguínea (Noro et al., 2013; Plaizier et al., 2001; Steele et al., 2012). Estes eventos desencadeiam uma cascata de respostas compensatórias resultando principalmente em menor consumo de matéria seca, laminite, comorbidades 
por redução da resposta imune, ruminite e abscessos hepáticos, os quais acarretam em menor desempenho e até o óbito dos animais (Krause \& Oetzel, 2006; Martens et al., 2012; Stone, 2004).

Posto isso se faz necessário detectar o mais precocemente possível esta síndrome, bem como sua magnitude, pois as perdas produtivas corresponderão à duração e à gravidade deste distúrbio (Tajik et al., 2011). Para isso o monitoramento precoce da SARA pode ser realizado por meio da mensuração do $\mathrm{pH}$ do líquido ruminal, coletado por ruminocentese, sondagem orogástrica ou por cânula em animais fistulados (Noro et al., 2013; Tajik et al., 2011).

Para animais não fistulados, a sondagem orogástrica é uma alternativa menos invasiva, de baixo custo e que permite colheres grandes volume de líquido ruminal, porém não permite o controle de qual saco ruminal está sendo coletado e ainda existe contaminação por saliva da amostra, questionando-se a qualidade da amostra obtida (Duffield et al., 2004). A técnica de ruminocentese é mais invasiva e limita o volume de liquido ruminal obtido, porém possui como vantagens baixo custo, obtenção de amostras desprovidas de contaminação por saliva e ainda possibilita a escolha do saco ruminal em que este líquido pode ser coletado (Duffield et al., 2004; Garrett et al., 1999). Estes dois últimos fatores são os principais responsáveis pela divergência de valores de $\mathrm{pH}$ encontrados quando as duas técnicas de colheita são aplicadas em um mesmo animal, o que garante maior credibilidade às amostras obtidas por ruminocentese (Broomfield \& Gibbs, 2011; Tajik et al., 2011). Embora a ruminocentese tenha sido a principal técnica de escolha para coleta de líquido ruminal, a maioria dos trabalhos não informa se ocorreram intercorrências após o procedimento (Gianesella et al., 2010; Khafipour et al., 2011; Kleen et al., 2009) e quando estas ocorreram, se limitaram a poucos animais e foram caracterizadas por abcessos locais de resolução espontânea ou ruminite local identificadas apenas no abate (Broomfield \& Gibbs, 2011; Noro et al., 2013; Tajik et al., 2011).

Geralmente estes pesquisadores utilizam vacas holandesas adultas, de temperamento calmo e com pouca deposição de gordura corporal quando comparados a bovinos de corte. Estes animais também eram submetidos a uma dieta de forragem e concentrado (Gianesella et al., 2010; Khafipour et al., 2011; Gianesella et al., 2010; Tajik et al., 2011), o que gera a dúvida se estes mesmos resultados podem ser aplicados a bovinos de engorda submetidos a dieta isenta de forragens.

Portanto, o objetivo deste trabalho foi avaliar a viabilidade da técnica de ruminocentes para bovinos de corte alimentados com dieta $100 \%$ concentrado isentas de forragem.

\section{Material e métodos}

Este experimento foi aprovado pelo comitê de ética em uso animal-CEUA-UNICENTRO-21/2015. O experimento foi realizado no Núcleo de Produção Animal (NUPRAN) junto ao Curso de Mestrado em Ciência Animal na Universidade Estadual do Centro-Oeste (UNICENTRO), localizada em Guarapuava, Paraná. Foram utilizados 32 novilhos não castrados (1/2 sangue Angus), com peso médio inicial de $350 \mathrm{~kg}$ e idade média inicial de 11 meses; sendo os animais previamente vermifugados e vacinados contra viroses respiratórias (V4J5 I, Biogen ${ }^{\circledR}$ ).

Os animais foram alojados em confinamento semicoberto, em piso de concreto com disposição de cocho com espaço livre de 1 metro por animal e bebedouro metálico automático. Inicialmente os animais receberam uma dieta composta por $1,2 \%$ do peso vivo (PV) dos animais de mistura concentrada (núcleo proteico, vitamínico e mineral: $15 \%$ + milho grão inteiro: $85 \%$ ) e silagem de milho à vontade por 15 dias; do décimo quinto ao décimo nono dia passou a ser fornecida 1,6\% do PV da mistura concentrada e silagem de milho à vontade; no vigésimo dia foi fornecida $1,8 \%$ PV e iniciada uma redução do fornecimento de volumoso de $25 \%$ ao dia em relação ao consumo do início do experimento até o vigésimo terceiro dia onde foi interrompido o fornecimento de alimento volumoso, disponibilizando somente a mistura de concentrado no cocho de forma "ad libitum" com ajustes diários de fornecimento.

A dieta $100 \%$ concentrado foi composta por uma mistura de $80 \%$ de grãos de milho inteiros e $20 \%$ de núcleo proteico-vitamínico-mineral, sendo fornecida de forma "ad libitum". A mistura concentrada foi elaborada na fábrica de rações comerciais da Cooperativa Agrária localizada na região de Entre Rios, Guarapuava, Paraná. 
$\mathrm{Na}$ preparação do concentrado proteico peletizado, foram utilizados os seguintes alimentos: farelo de soja, farelo de trigo, radícula de malte, calcário calcítico, fosfato bicálcico, uréia pecuária, premix vitamínico e mineral, sal comum, monensina sódica $\left(75 \mathrm{mg} \mathrm{kg}^{-1}\right)$ e virginiamicina $\left(75 \mathrm{mg} \mathrm{kg}^{-1}\right)$. Desta forma o núcleo proteico utilizado na dieta apresentou teores médios percentuais de MS de 90,22\%, PB de $42,23 \%$, EE de 2,59\%, FDN de 24,61\%, FDA de 12,28\%, MM de 16,31\%, cálcio (Ca) de 2,77\%, fósforo $(\mathrm{P})$ de $1,11 \%$, potássio $(\mathrm{K})$ de 1,57\% e magnésio $(\mathrm{Mg})$ de $0,48 \%$, com base na matéria seca total.

Para monitoramento da SARA, o líquido ruminal foi colhido, em dois momentos, a saber: no primeiro momento, 15 dias após a chegada dos animais a instalação (M0) e após a adaptação da dieta $100 \%$ concentrado e isenta de forragem (M1), 24 dias após a chegada dos animais.

Para a colheita do líquido ruminal, os animais foram submetidos a jejum prévio de 4 horas e contidos em tronco de contenção. Após prévia tricotomia e anti-sepsia com iodo poilvidona e álcool 70\%, colheuse líquido ruminal do saco ventro caudal do rúmen, por ruminocentese realizada com cateter $14 \mathrm{G}$ acoplado a uma seringa de $20 \mathrm{ml}$, entre 12 a $15 \mathrm{~cm}$ caudal a junção costocondral da última costela, na altura da crista patelar, tal qual descrito por Tajik et al. (2011), sem previa anestesia local. Após a punção, o cateter foi removido e o local limpo com álcool iodado. Avaliou-se a dificuldade de obtenção do líquido ruminal por meio de reação do animal e por número de tentativas para obtenção do material. Observou-se também as características físicas e químicas relacionadas a volume, coloração e viscosidade por inspeção visual e o pH do líquido ruminal, aferido imediatamente após a colheita, por meio de $\mathrm{pH}$ metro digital.

Avaliou-se também as intercorrências da ruminocentese diariamente durante sete dias após a coleta, verificando-se os parâmetros vitais constituídos por frequência cardíaca mediante auscultação das bulhas cardíacas durante um minuto, frequência respiratória, mediante inspeção da movimentação do gradil costal durante um minuto, movimentos ruminal, contando-se apenas os movimentos completos mediante auscultação do flanco esquerdo durante cinco minutos, temperatura corporea, mediante termometria retal; redução no consumo alimentar baseado nas sobras de alimento no cocho, pesagem do animal no dia de cada colheita e sete dias após, em balança digital após jejum prévio de 4 horas e reações locais verificado por nodulações ou abcessos no local da punção.

A análise estatística dos dados foi realizada utilizando-se o software estatístico Instat Graphpad. Para a avaliação, calculou-se as médias referentes ao $\mathrm{pH}$ ruminal, parâmetros vitais, ganho de peso e consumo de matéria seca durante os sete dias após cada colheita. As médias dos resultados obtidos, foram submetidos ao teste $\mathrm{T}$ paramétrico. Os resultados relacionados as reações locais após a punção foram transformadas em escore e analisados pelo teste do qui-quadrado, sendo expressos como frequência de animais com a alteração. Para todos os resultados, foram considerados como significantes os dados que apresentarem nível de significância menor ou igual a $5 \%(\mathrm{P} \leq 0,05)$.

\section{Resultados}

Foi possível obter líquido ruminal de 31 animais dos 32 nos dois momentos, diferindo o animal cuja coleta foi improdutiva em cada momento (Tabela 1). No M0 28/32 (87,5\%) dos animais reagiram à introdução do cateter na pele e musculatura, dificultando a colheita. Já no M1, apenas $2 / 32(6,25 \%)$ reagiram à introdução do cateter na pele e musculatura, pois os animais já estavam mais habituados à contenção. No entanto, estavam maiores, mais pesados e com aumento da espessura abdominal. Assim a exposição do local de punção pelas aberturas do tronco de contenção era mais limitada e apesar da introdução do cateter na pele e musculatura ser realizada com maior facilidade, o cateter nem sempre atingia o saco ruminal, precisando ser redirecionado em 27/32 animais (84,4\%), o que gerava incômodo no animal.

Mediante as dificuldades encontradas durante as punções, os volumes obtidos de líquido ruminal variaram entre 0,5 a $1 \mathrm{ml} \mathrm{em}$ todos os animais nos dois momentos. No M0 o líquido ruminal apresentou cor verde oliva, viscoso, com ausência de contaminação por sangue em todos os animais (100\%) e com valores de $\mathrm{pH}$ de 7,0 (100\%). No M1, o líquido era de cor esbranquiçada a leitosa em 24/31 animais $(77,4 \%)$ e em $7 / 31$ animais $(22,6 \%)$ as amostras tiveram contaminação por sangue. Estava aquoso em todos os animais (100\%) e o valor médio do $\mathrm{pH}$ foi 6,0 .

Após o M0 não houve presença de abscessos ou reações locais, bem como não foi observada redução no consumo alimentar de em nenhum dos animais. No entanto no M1 todos os animais apresentaram 
algum tipo de reação local, $9 / 32(28,1 \%)$ aumento de volume firme menor que $5 \mathrm{~cm}$ no local da punção, $21 / 32$ animais $(65,6 \%)$ apresentaram abscessos entre 5 a $15 \mathrm{~cm}$ e 2/32 animais $(6,3 \%)$ apresentaram abcessos maiores que $20 \mathrm{~cm}$, necessitando de drenagem do abscesso, e uso de anti-inflamatório e antibiótico (tabela 1). Apenas 2/32 (6,25\%) animais apresentaram redução de consumo alimentar nos 3 primeiros dias após a punção, sem diferença estatística entre o consumo após punção no M0 e M1, e nenhum animal apresentou alterações em frequências cardíacas, respiratória, movimento ruminal ou temperatura corporal (Tabela 2).

Tabela 1. Facilidade de coleta, características física e química do líquido ruminal e reações locais após a ruminocentese de novilhos de corte alimentados com dieta composta e isenta de forragem.

\begin{tabular}{|c|c|c|}
\hline Parâmetros & M0 & M1 \\
\hline \multicolumn{3}{|l|}{ Facilidade de obtenção do líquido ruminal } \\
\hline Coletas produtivas & 31 & 31 \\
\hline Volume obtido (mL) & $05-1 \mathrm{ml}$ & $05-1 \mathrm{ml}$ \\
\hline Animais que reagiram a introdução do cateter (\%) & 87,5 & 6,25 \\
\hline Animais que precisaram de nova punção para obtenção do líquido ruminal (\%) & 0 & 84,4 \\
\hline \multicolumn{3}{|l|}{ Características do líquido ruminal } \\
\hline Animais com líquido ruminal verde oliva (\%) & 100 & 0 \\
\hline Animais com líquido ruminal esbranquiçado (\%) & 0 & 77,4 \\
\hline Animais com líquido ruminal avermelhado (\%) & 0 & 22,6 \\
\hline Animais com líquido ruminal viscoso (\%) & 100 & 0 \\
\hline Animais com líquido ruminal aquoso (\%) & 0 & 100 \\
\hline pH do líquido ruminal - média (SEM) & $7,0(0,1)$ & $6(0,4)$ \\
\hline \multicolumn{3}{|l|}{ Reações locais após ruminocentese } \\
\hline Animais com reações locais ausentes (\%) & 100 & 0 \\
\hline Animais com nódulos ou abscessos menor que $5 \mathrm{~cm}(\%)$ & 0 & 28,1 \\
\hline Animais com nódulos ou abscessos entre 5 a 15 cm (\%) & 0 & 65,6 \\
\hline Animais com nódulos ou abscessos maior que $20 \mathrm{~cm}(\%)$ & 0 & 6,25 \\
\hline $\mathrm{P} *$ & \multicolumn{2}{|c|}{0,0001} \\
\hline
\end{tabular}

*líquido ruminal coletado aos 15 dias da chegada dos animais ao confinamento (M0) em dieta com presença de volumoso e aos 24 dias (M1) em dieta 100\% concentrada isenta de forragem. *Teste do qui quadrado.

Os animais foram abatidos 104 dias depois de chegarem às instalações. No abate notou-se que 11/32 $(34,4 \%)$ animais ainda apresentavam reação inflamatória no local da punção, com persistência do abscesso e condenação da região.

Tabela 2. Parâmetros vitais e consumo alimentar de novilhos de corte alimentados com dieta composta e isenta de forragem após a ruminocentese

\begin{tabular}{lcccc}
\hline Parâmetros & M0 & M1 & SEM & P \\
\hline FC $($ bpm) & 100 & 103 & 10 & 0,60 \\
FR (mrm) & 63 & 68 & 15 & 0,70 \\
MR completos (mr/5 min) & 2 & 1,5 & 1 & 0,8 \\
T. $\left({ }^{\circ}\right.$ C) & 38,8 & 39,0 & 0,4 & 0,8 \\
Consumo matéria seca(kg/dia) & 7,09 & 6,82 & 0,22 & 0,56 \\
Ganho de peso diário $(\mathrm{kg} / \mathrm{d})$ & 1,15 & 1,12 & 0,06 & 0,89 \\
\hline
\end{tabular}

Valores expressos em média durante os sete dias de avaliação a pós cada punção. SEM- erro padrão da média-FC- frequência cardíaca, FR frequência respiratória, MR- movimentos ruminais, T. ${ }^{\circ} \mathrm{C}$ temperatura retal

\section{Discussão}

Estudos anteriores relataram pouca reação após a ruminocentese, como Kleen et al. (2009) que reportaram 5,5\% de incidência de abscessos. Duffield et al. (2004) que relataram abscessos de apenas $1-2 \mathrm{~cm}$ de diâmetros. Noro et al. (2013) que relataram apenas $4 \%$ dos animais com pequenos aumentos de volume local de 1 a $1,5 \mathrm{~cm}$, os quais regrediram e desapareceram sem qualquer intervenção. 
Gianesella et al. (2010) citaram mínimas alterações locais e Tajik et al. (2011) que mencionaram presença de pequenas inflamações locais e de apenas um caso de abcesso de $1 \mathrm{~cm}$ de diâmetro que curou espontaneamente sem necessidade de intervenção.

O presente estudo relata uma porcentagem maior, na qual todos os animais apresentaram reação local e 71,9\% dos animais apresentaram abscessos maiores que $5 \mathrm{~cm}$ no M1, que não regrediram com o passar do tempo. Possivelmente esta diferença ocorreu porque o presente trabalho foi feito em bovinos de engorda, diferentemente dos autores supracitados que realizaram seus experimentos com vacas leiteiras que possuem temperamento mais dócil e menor espessura de gordura abdominal (Arango et al., 2002), o que facilita a colheita e minimiza o estresse (Duffield et al., 2004) gerando menor injúria local e consequentemente poucas tentativas para coletar o líquido ruminal.

Apesar de no M0 haver dificuldade em realizar a ruminocentese devido à reação à introdução do cateter na pele e musculatura, nenhum animal apresentou reação local neste momento. Tais achados diferiram daqueles obtidos por Gianesella et al. (2010) que não observaram resistência dos animais à ruminocentese e obtiveram maiores volumes de líquido e não registraram reações locais.

No M1, os animais já haviam crescido, o que dificultou a exposição do local de punção da ruminocentese, situação citada por Noro et al. (2013), mas que no trabalho prévio não limitou nem o volume de liquido ruminal, nem favoreceu a ocorrência de reações inflamatórias após a punção, diferentemente do encontrado no presente trabalho.

Como em ambas as colheitas o volume de líquido ruminal foi muito abaixo dos $3 \mathrm{~mL}$ que foram encontrados nos prévios trabalhos de Noro et al. (2013) e Kleen et al. (2013). Ainda, foi possível detectar que a mudança da dieta entre o M0 e o M1 promoveu acidificação do $\mathrm{pH}$, o que pode ter corroborado para o processo inflamatório local (Khafipour et al., 2011; Mialon et al., 2012). Em situações de maior acidez ruminal há maior na fluidez do líquido ruminal, o que de fato foi verificado no presente trabalho, o que pode ter favorecido o extravasamento do líquido para o tecido subcutâneo no M1 inoculando na região grande quantidade de microrganismos, o que levou à formação dos abscessos posteriores, tal qual relatado por Mialon et al. (2012).

Partindo do pressuposto que as reações locais após a ruminocentese foram causadas por extravasamento de líquido ruminal para a região subcutânea, sugere-se que novos trabalhos sejam realizados para verificar a viabilidade da coleta do líquido ruminal por meio de punção do saco caudo dorsal ruminal como uma alternativa com menor complicações para bovinos de corte confinados com dieta de $100 \%$ de concentrado isenta de forragem.

\section{Conclusão}

Conclui-se que a técnica de ruminocentese do saco ventro caudal idealizada para vacas leiteiras não foi adequada para bovinos de engorda submetidos à dieta $100 \%$ concentrado isenta de forragem, por desencadear reação local e abscessos em todos os animais deste experimento.

\section{Agradecimentos}

Os autores agradecem à Coordenação de Aperfeiçoamento de Pessoal de Nível Superior (CAPES), pela bolsa de mestrado de Angela M. Reck.

\section{Referências Bibliográficas}

Arango, J. A., Cundiff, L. V, \& Van Vleck, L. D. (2002). Breed comparisons of Angus, Charolais, Hereford, Jersey, Limousin, Simmental, and South Devon for weight, weight adjusted for body condition score, height, and body condition score of cows. Journal of Animal Science, 80(12), 31233132.

Berchielli, T. T., Pires, A. V, Oliveira, S. G., \& FUNEP. (2011). Nutrição de Ruminantes (Issue 2th ed.). FUNEP.

Broomfield, A. M., \& Gibbs, S. J. (2011). The effect of three techniques of rumen $\mathrm{pH}$ assessment on measured $\mathrm{pH}$ of rumen fluid and digesta in an in vitro artificial rumen system. Proceedings of the New Zealand Society of Animal Production, 71, 9-12. 
Cumby, J. L., Plaizier, J. C., Kyriazakis, I., \& McBride, B. W. (2001). Effect of subacute ruminal acidosis on the preference of cows for pellets containing sodium bicarbonate. Canadian Journal of Animal Science, 81(1), 149-152. https://doi.org/10.4141/A00-099

Duffield, T., Plaizier, J. C., Fairfield, A., Bagg, R., Vessie, G., Dick, P., Wilson, J., Aramini, J., \& McBride, B. (2004). Comparison of techniques for measurement of rumen $\mathrm{pH}$ in lactating dairy cows. Journal of Dairy Science, 87(1), 59-66. https://doi.org/http://dx.doi.org/10.3168/jds.S00220302(04)73142-2

Garrett, E. F., Pereira, M. N., Nordlund, K. V, Armentano, L. E., Goodger, W. J., \& Oetzel, G. R. (1999). Diagnostic methods for the detection of subacute ruminal acidosis in dairy cows. Journal of Dairy Science, 82(6), 1170-1178.

Gianesella, M., Morgante, M., Stelletta, C., Ravarotto, L., Giudice, E., \& Van Saun, R. J. (2010). Evaluating the effects of rumenocentesis on health and performance in dairy cows. Acta Veterinaria Brno, 79(3), 459-468.

Khafipour, E., Plaizier, J. C., Aikman, P. C., \& Krause, D. O. (2011). Population structure of rumen Escherichia coli associated with subacute ruminal acidosis (SARA) in dairy cattle. Journal of Dairy Science, 94(1), 351-360. https://doi.org/http://dx.doi.org/10.3168/jds.2010-3435

Kleen, J L, Hooijer, G. A., Rehage, J., \& Noordhuizen, J. P. T. M. (2009). Subacute ruminal acidosis in Dutch dairy herds. Veterinary Record, 164(22), 681-683.

Kleen, Joachim L, Upgang, L., \& Rehage, J. (2013). Prevalence and consequences of subacute ruminal acidosis in German dairy herds. Acta Veterinaria Scandinavica, 55(1), 1-6.

Krause, K. M., \& Oetzel, G. R. (2006). Understanding and preventing subacute ruminal acidosis in dairy herds: A review. Animal Feed Science and Technology, 126(3), 215-236.

Li, S., Khafipour, E., Krause, D. O., Kroeker, A., Rodriguez-Lecompte, J. C., Gozho, G. N., \& Plaizier, J. C. (2012). Effects of subacute ruminal acidosis challenges on fermentation and endotoxins in the rumen and hindgut of dairy cows. Journal of Dairy Science, 95(1), 294-303. https://doi.org/http://dx.doi.org/10.3168/jds.2011-4447

Martens, H., Rabbani, I., Shen, Z., Stumpff, F., \& Deiner, C. (2012). Changes in rumen absorption processes during transition. Animal Feed Science and Technology, 172(1-2), 95-102. https://doi.org/http://dx.doi.org/10.1016/j.anifeedsci.2011.12.011

Mialon, M.-M., Deiss, V., Andanson, S., Anglard, F., Doreau, M., \& Veissier, I. (2012). An assessment of the impact of rumenocentesis on pain and stress in cattle and the effect of local anaesthesia. The Veterinary Journal, 194(1), 55-59.

Monteschio, J. O., Vargas Junior, F. M., Almeida, F. L. A., Pinto, L., Kaneko, I. N., Almeida, A. A., Freitas, L. W., Alves, S. P., Bessa, R. J. B., \& Prado, I. N. (2019). The effect of encapsulated active principles (eugenol, thymol and vanillin) and clove and rosemary essential oils on the structure, collagen content, chemical composition and fatty acid profile of Nellore heifers muscle. Meat Science, 155, 27-35. https://doi.org/https://doi.org/10.1016/j.meatsci.2019.04.019.

Noro, M., Sepúlveda, P., Cárdenas, F., Chihuailaf, R. H., \& Wittwer, F. (2013). Rumenocentesis dorsomedial: un procedimiento seguro para la obtención de líquido ruminal en vacas lecheras a pastoreo. Archivos de Medicina Veterinaria, 45(1), 25-31.

Ornaghi, M. G., Passetti, R. A. C., Torrecilhas, J. A., Mottin, C., Vital, A. C. P., Guerrero, A., Sañudo, C., Campo, M. M., \& Prado, I. N. (2017). Essential oils in the diet of young bulls: Effect on animal performance, digestibility, temperament, feeding behaviour and carcass characteristics. Animal Feed Science and Technology, 234, 274-283. https://doi.org/10.1016/j.anifeedsci.2017.10.008

Plaizier, J. C., Keunen, J. E., Walton, J. P., Duffield, T. F., \& McBride, B. W. (2001). Effect of subacute ruminal acidosis on in situ digestion of mixed hay in lactating dairy cows. Canadian Journal of Animal Science, 81(3), 421-423. https://doi.org/10.4141/A00-106

Rivaroli, D. C., Ornaghi, M. G., Mottin, C., Prado, R. M., Ramos, T. R., Guerrero, A., Jorge, A. M., \& Prado, I. N. (2017). Essential oils in the diet of crossbred (1/2 Angus vs. $1 / 2$ Nellore) bulls finished in feedlot on animal performance, feed efficiency and carcass characteristics. Journal of Agricultural Science, 9(10), 205-212. https://doi.org/http://dx.doi.org/10.5539/jas.v9n10p205-212. 
Steele, M. A., Dionissopoulos, L., AlZahal, O., Doelman, J., \& McBride, B. W. (2012). Rumen epithelial adaptation to ruminal acidosis in lactating cattle involves the coordinated expression of insulin-like growth factor-binding proteins and a cholesterolgenic enzyme. Journal of Dairy Science, 95(1), 318327. https://doi.org/http://dx.doi.org/10.3168/jds.2011-4465

Stone, W. C. (2004). Nutritional Approaches to Minimize Subacute Ruminal Acidosis and Laminitis in Dairy Cattle. Journal of Dairy Science, 87, Supple(0), E13-E26. https://doi.org/http://dx.doi.org/10.3168/jds.S0022-0302(04)70057-0

Tajik, J., Nadalian, M. G., Raoofi, A., Mohammadi, G. R., \& Bahonar, A. R. (2011). Evaluation of rumenocentesis practicability as a routine diagnostic technique in veterinary practice. Veterinarski Arhiv, 81(5), 557-561.

Van Soest, P. J. (1994). Nutritional ecology of the ruminant (Vol. 1, Issue 2). Cornell University Press.

Zebeli, Q., \& Ametaj, B. N. (2009). Relationships between rumen lipopolysaccharide and mediators of inflammatory response with milk fat production and efficiency in dairy cows. Journal of Dairy Science, 92(8), 3800-3809. https://doi.org/http://dx.doi.org/10.3168/jds.2009-2178

Zebeli, Q., Aschenbach, J. R., Tafaj, M., Boguhn, J., Ametaj, B. N., \& Drochner, W. (2012). Invited review: Role of physically effective fiber and estimation of dietary fiber adequacy in high-producing dairy cattle. Journal of Dairy Science, 95(3), 1041-1056. https://doi.org/http://dx.doi.org/10.3168/jds.2011-4421

Zebeli, Q., \& Metzler-Zebeli, B. U. (2012). Interplay between rumen digestive disorders and dietinduced inflammation in dairy cattle. Research in Veterinary Science, 93(3), 1099-1108. https://doi.org/http://dx.doi.org/10.1016/j.rvsc.2012.02.004

Recebido: 01 de agosto, 2019.

Aprovado: 21 de outubro, 2019.

Publicado: 23 de abril, 2020.

Licenciamento: Este artigo é publicado na modalidade Acesso Aberto sob a licença Creative Commons Atribuição 4.0 (CC-BY 4.0), a qual permite uso irrestrito, distribuição, reprodução em qualquer meio, desde que o autor e a fonte sejam devidamente creditados. 